\title{
Fluxograma multiprofissional para atendimento de intoxicações agudas por agrotóxicos na atenção primária à saúde
}

\author{
Multiprofessional flowchart for care of acute intoxications by agrotoxic in primary health care \\ Diagrama de flujo multiprofesional para la atención del envenenamiento agudo por agrotoxicos en la \\ atención primaria de salud
}

\begin{abstract}
Adriane Karal ${ }^{1}$ (1)
Dara Montag Portaluppi ${ }^{2}$

Denise Antunes de Azambuja Zocche ${ }^{1,3}$ (1)

Leila Zanatta ${ }^{1,3}$ (1)

1. Universidade do Estado de Santa Catarina, Programa de Pós-Graduação Stricto Sensu em Enfermagem. Chapecó, SC, Brasil.
\end{abstract}

2. Universidade do Estado de Santa Catarina, Curso de Graduação em Enfermagem.

Chapecó, SC, Brasil.

3. Universidade do Estado de Santa Catarina, Departamento de Enfermagem. Chapecó, SC, Brasil.

\section{Autor correspondente:}

Adriane Karal.

E-mail: adri.karal@hotmail.com

Recebido em 17/02/2021.

Aprovado em 26/09/2021.

DOl:https://doi.org/10.1590/2177-9465-EAN-2021-0015

\section{Resumo}

Objetivo: construir e validar um fluxograma de atendimento multiprofissional para casos de intoxicações agudas por agrotóxicos na Atenção Primária à Saúde. Método: estudo metodológico, realizado em duas etapas: produção-construção e validação do conteúdo. A construção do fluxograma foi realizada com base na literatura, abordando a temática agrotóxicos, e entrevista coletiva envolvendo 19 profissionais da Atenção Primária à Saúde de um município catarinense, em dezembro de 2018. 0 processo de validação foi realizado por meio de questionário do Google Forms, por sete juízes. Utilizou-se o Percentual de Concordância e o Índice de Validade de Conteúdo. Resultados: o fluxograma apresentou validade de conteúdo global igual a 0,97, tendo os oito primeiros itens apresentado valor máximo e aprovação pelo Conselho Municipal de Saúde. Conclusão: o fluxograma passou a ser utilizado como instrumento tecnológico que orienta e qualifica os atendimentos dos casos de intoxicações agudas por agrotóxicos. Implicações para a prática: a utilização do fluxograma na assistência ao paciente, suspeito ou intoxicado por agrotóxico, constitui-se como ferramenta de gestão do cuidado, permitindo que a equipe de saúde atue de forma harmônica, além de que as ações da assistência sejam sistematizadas e, consequentemente, se obtenha qualidade do serviço prestado.

Palavras-chave: Enfermagem; Agroquímicos; Atenção Primária à Saúde; Fluxo de Trabalho; Equipe de Assistência ao Paciente.

\section{Abstract}

Objective: To construct and validate a multiprofessional care flowchart for acute pesticide intoxication cases in Primary Health Care. Method: This is a methodological study, carried out in two stages: production-construction and content validation. The flowchart construction was carried out based on scientific literature, addressing the topic of pesticides, and a press conference involving 19 Primary Health Care professionals in a municipality in Santa Catarina, in December 2018. The validation process was carried out through a Google Forms questionnaire, by seven judges. The Percentage of Agreement and the Content Validity Index were used. Results: The flowchart had a global content validity of 0.97 , with the first eight items having a maximum value and approval by the Municipal Health Council. Conclusion: The flowchart started to be used as a technological tool that guides and qualifies the care of acute pesticide intoxications cases. Implications for practice: The flowchart use in care for patients suspected or intoxicated by pesticides is a care management tool, allowing the health team to act harmoniously, in addition to assistance actions systematized and, consequently, obtain the quality of the service provided.

Keywords: Nursing; Agrochemicals; Primary Health Care; Workflow; Patient Care Team.

\section{RESUMEN}

Objetivo: construir y validar un diagrama de flujo de atención multiprofesional para casos de intoxicación aguda por plaguicidas en Atención Primaria de Salud. Método: estudio metodológico, realizado en dos etapas: producción-construcción y validación de contenido. La construcción del diagrama de flujo se llevó a cabo a partir de la literatura que aborda el tema plaguicidas, y una entrevista colectiva a 19 profesionales de Atención Primaria de Salud de un municipio de Santa Catarina, en diciembre de 2018. El proceso de validación se realizó a través de un cuestionario de Google Forms, por siete jueces. Se utilizaron el porcentaje de acuerdo y el índice de validez de contenido. Resultados: el diagrama de flujo tuvo una validez de contenido global de 0.97 , mostrando los primeros ocho ítems el valor máximo y la aprobación del Consejo Municipal de Salud. Conclusión: el diagrama de flujo pasó a ser utilizado como un instrumento tecnológico que orienta y califica la atención en casos de intoxicación aguda por plaguicidas. Implicaciones para la Practica: el uso del diagrama de flujo en el cuidado de pacientes sospechosos o intoxicados por pesticidas es una herramienta de gestión de la atención, permitir que el equipo de salud actúe en armonía, que se sistematicen las acciones asistenciales y consecuentemente se obtiene calidad de servicio.

Palabras claves: Enfermería; Agroquímicos; Atención Primaria de Salud; Flujo de Trabajo; Grupo de Atención al Paciente. 


\section{INTRODUÇÃO}

A carta de Ottawa para promoção da saúde, produzida na década de 1986, reconhece diversos fatores determinantes do processo saúde-doença e ressalta a promoção da saúde, como processo que permite, aos indivíduos, realizar ações que promovam a melhoria da qualidade de vida e de saúde. A carta define saúde em uma visão ampliada, que passa a ser entendida como recurso para a vida, envolvendo o bem-estar físico, mental e social ${ }^{1}$. Ainda, reforça a importância da criação de ambientes saudáveis, a proteção do meio ambiente e a conservação dos recursos, bem como da reorientação dos serviços de saúde.

Apesar de o contexto mundial ter se modificado desde a primeira conferência para a promoção da saúde, esses aspectos foram inseridos nas demais e, mais recentemente, na defesa da saúde, em todas as políticas, como parte integrante de outros setores que podem produzir efeitos sobre a saúde e meio ambiente $^{2}$. Ressalta-se também o reconhecimento da saúde e do bem-estar como essência para o alcance dos objetivos pactuados na Agenda 2030, identificando o potencial de promover a saúde em relação ao desenvolvimento sustentável e de envolver a sociedade no processo de desenvolvimento da saúde para a garantia de uma vida saudável ${ }^{3}$.

Dessa forma, a percepção de saúde envolve fatores políticos, econômicos, culturais, sociais e, não menos importante, as condições ambientais ${ }^{1}$. No Brasil, frequentemente, os indivíduos estão expostos a substâncias químicas, como os agrotóxicos, também denominados agroquímicos, presentes em ambientes diversos, como domicílios, locais de trabalho, escolas e comunidade em geral, gerando contaminação do ar, água e solo ${ }^{4}$. O que implica na existência de riscos de danos à saúde, devido aos efeitos deletérios dos agrotóxicos ${ }^{5}$, principalmente, aos agricultores que, raramente, utilizam Equipamentos de Proteção Individual $(\mathrm{EPI})^{6}$. Em 1989, foi lançada a Lei $\mathrm{n}$ ㅇ 7.802 , conhecida como a Lei dos agrotóxicos, a qual vem regulamentando aspectos da produção, comercialização, registro dos agrotóxicos, entre outros. Dentro das políticas públicas saudáveis, percebe-se que, na conjuntura, essa Lei foi uma conquista adquirida para a conservação de recursos naturais e à proteção do meio ambiente, consequentemente, da saúde humana?.

O Brasil está entre os países que mais utilizam agrotóxicos e para cultivar grandes extensões e exportar matéria-prima, o modelo hegemônico de produção agrícola, no Brasil, tem por base a monocultura de espécies geneticamente modificadas, sustentado pelo uso de grandes quantidades desses produtos ${ }^{7}$. Em consonância, no ano de 2010, o mercado nacional movimentou $19 \%$ do mercado global de agrotóxicos, aproximadamente US\$7,3 bilhões, considerado o maior mercado mundial de agrotóxicos $^{8}$.

Nesse contexto, e levando em conta o cenário atual do Brasil, tem-se ainda o desafio de alcançar os objetivos da agenda, previstos para 2020, no que se refere ao manejo, ambientalmente, saudável dos agrotóxicos e seus resíduos, além da redução de liberação no ambiente, para minimizar os impactos negativos ${ }^{9}$. Tendo em vista que as commodities agrícolas são um dos principais pilares da economia brasileira, os benefícios do uso de agrotóxicos devem estar em equilíbrio com os potenciais riscos. Entretanto, os efeitos na saúde, principalmente, ao longo prazo, decorrentes da exposição ocupacional a pesticidas, no Brasil, ainda são incipientes, fazendose necessários mais estudos para suprir essa lacuna ${ }^{6}$.

Segundo dados do Sistema de Informações de Agravos de Notificação (Sinan), no período de 2007 a 2015, o Brasil teve um aumento no número de notificações de intoxicação por agrotóxicos, com acréscimo de $139 \%$ e com um total acumulativo de 84.206 casos notificados. Essa alta no número de casos é, provavelmente, resultado do aumento de comercialização dos agrotóxicos, de sua toxicidade intrínseca, e da melhoria da vigilância e da assistência à saúde em identificar, diagnosticar e notificar os casos ${ }^{10}$.

Frente a esse contexto, no âmbito dos serviços de saúde, a Atenção Primária a Saúde (APS), no Brasil, denominada de Atenção Básica $(A B)$, apresenta-se como ponto preferencial de entrada. É, também, componente da Rede de Atenção à Saúde (RAS), primordial ao prestar cuidados fundamentais com ações de promoção, prevenção, tratamento, vigilância em saúde ${ }^{11}$, envolvendo situações relativas à exposição, bem como, a intoxicação por agrotóxico.

Do ponto de vista da integralidade, a APS procura atender as necessidades dos indivíduos, famílias e comunidades do seu território de abrangência, e considera os determinantes, os riscos e os danos à saúde, nos processos de trabalho da equipe multidisciplinar que a constitui. Além disso, atua coordenando o cuidado nesse nível de atenção e direciona a oferta de serviços em outros pontos da rede ${ }^{11}$

O enfermeiro, inserido nas equipes multidisciplinares da APS, produz diversas ações de saúde, mediante conhecimento técnico e científico. Como parte de suas atividades, desenvolve o cuidado de enfermagem para promoção e prevenção de situações como as intoxicações, ao realizar orientações, atividades de educação em saúde referente aos riscos à saúde e aos perigos da exposição, bem como nos casos de intoxicações, atuando, diretamente, na coleta de dados, no reconhecimento de sinais e sintomas, para o diagnóstico e orientação do tratamento, no intuito de diminuir os riscos de morbimortalidade ${ }^{12}$.

As intoxicações agudas por agrotóxicos decorrem de uma única exposição ou de sucessivas exposições em um curto período, podendo causar efeitos imediatos, identificados a partir de vertigens, náuseas, vômitos, cefaleia, tosse, febre e calafrios, gosto amargo na boca, dor epigástrica, cólicas abdominais, diarreia e prurido pelo corpo ${ }^{13}$. O diagnóstico, a partir desses sintomas, nem sempre é fácil, visto a inespecificidade dos mesmos e, portanto, conhecer o contexto em que se encontra o usuário do serviço de saúde é essencial.

Estudo recente aponta aumento significativo da demanda de atendimento de casos de intoxicação por agrotóxicos para a enfermagem e a necessidade de reorganização do serviço e a qualificação profissional ${ }^{14}$. Para tanto, o uso de tecnologias no processo de trabalho do enfermeiro, vem no intuito de facilitar suas ações como integrante e atuante da equipe, tornando-as mais resolutivas ${ }^{15}$ 
Sejam elas materiais ou não, as tecnologias têm potencial para intervir em determinadas situações. Um exemplo, são os fluxogramas como instrumento tecnológico, que otimizam os atendimentos e permitem visualizar o fluxo de trabalho e os momentos de produção de cuidado ${ }^{16}$. Percebe-se que a construção e a validação de instrumentos têm sido, cada vez mais, presentes nas pesquisas, em decorrência de muitos dos profissionais de enfermagem reconhecerem a necessidade de utilizar esses instrumentos na prática diária ${ }^{17}$.

Diante desse contexto, tem-se a seguinte questão norteadora do estudo: uma tecnologia em saúde, construída em conjunto com a equipe multiprofissional, pode auxiliar os profissionais da APS para qualificar o atendimento dos usuários com intoxicações agudas por agrotóxicos? A partir dessa inquietação pretendeu-se, neste estudo, construir e validar um fluxograma de atendimento multiprofissional para casos de intoxicações agudas por agrotóxicos na atenção primária à saúde.

\section{MÉTODO}

Trata-se de um estudo metodológico, desenvolvido em duas etapas: a produção-construção do fluxograma de atendimento multiprofissional para casos de intoxicações agudas por agrotóxicos na APS e a validação desse material por profissionais que atuam na APS de um município do Extremo-Oeste do estado de Santa Catarina. As pesquisas metodológicas têm como foco o desenvolvimento, avaliação e aperfeiçoamento de instrumentos e estratégias metodológicas ${ }^{18}$.

No processo de produção-construção do fluxograma (etapa 1), buscou-se realizar um diagnóstico situacional, identificando as práticas de saúde desenvolvidas na APS, frente ao indivíduo intoxicado por agrotóxico e as fragilidades desse atendimento. Essas informações foram apontadas pelos profissionais participantes da pesquisa, por meio de entrevista coletiva, na qual utilizou-se um roteiro com seis questões semiestruturadas. As entrevistas coletivas foram áudio gravadas e transcritas no Microsoft Word para posterior análise. Os dados obtidos durante a pesquisa foram arquivados pela pesquisadora e serão mantidos pelo período de cinco anos e depois inutilizados.

A coleta de dados, por meio da entrevista coletiva, foi realizada com cada uma das cinco equipes de Saúde da Família (eSF) e com uma equipe de Atenção Básica (eAB) de Unidade Básica de Saúde (UBS) do município. Para participar da pesquisa, foram considerados profissionais enfermeiros, médicos e técnicos de enfermagem, que estavam atuando há, pelo menos, um ano na APS do município e que estiveram presentes no momento da entrevista coletiva, previamente agendada. Os profissionais que aceitaram participar do estudo assinaram o Termo de Consentimento Livre e Esclarecido. A coleta de dados foi realizada durante o mês de dezembro de 2018 e dela participaram 19 do total de 26 profissionais.

A identificação de cada participante, durante a pesquisa, foi preservada, sendo realizada com as iniciais $\mathrm{E}$ (Enfermeiro), TE (Técnico de Enfermagem) e M (Médico), sendo acompanhadas de numeração: $E(1), E(2), T E(1), T E(2), M(1), M(2)$, respectivamente.

Para a produção-construção do fluxograma, também foi realizada uma revisão integrativa da literatura, na qual foram analisadas as principais publicações sobre intoxicação por agrotóxicos na APS, obtidas nas bases de dados da Coordenação de Aperfeiçoamento de Pessoal de Nível Superior (Capes) e da Biblioteca Virtual em Saúde (BVS). Utilizou-se o referencial teórico de construção de fluxograma proposto por Silva e Silvino ${ }^{19}$. A estrutura do fluxograma segue um conjunto de símbolos que representam as etapas do processo, as pessoas, a sequência das ações e a circulação dos dados e dos documentos, evidenciando origem, processo e destino da informação. O fluxograma horizontal, apresentado neste estudo, constitui-se em instrumento gráfico que enfatiza as pessoas envolvidas em determinada rotina, com o objetivo de descrever os eventos de forma detalhada ${ }^{19}$.

Para a elaboração desse fluxograma, considerou-se quatro símbolos: oval; retângulo; losango; e a seta fina e contínua. O símbolo oval tem o significado terminal ou terminador e representa início, parada ou fim do processo; o retângulo tem significado de operação/ processo e representa ação; o losango, com significado de ponto de decisão, escolha de alternativas; e a seta fina e contínua, utilizada para indicar o sentido do fluxo de documentos e processos ${ }^{19}$.

Os profissionais envolvidos no estudo receberam a primeira versão do fluxograma de atendimento multiprofissional para os casos de intoxicação aguda por agrotóxicos durante capacitação sobre agrotóxicos, que foi realizada pela pesquisadora e voltada aos profissionais da APS. Os profissionais foram orientados a realizar a análise do fluxograma e propor sugestões para adequações do mesmo e posterior validação.

Para a validação do fluxograma (etapa 2), utilizou-se o conceito de validade de conteúdo, com instrumento do tipo questionário, baseado em julgamento de adequação de um conjunto de itens com relação ao conteúdo. Os dados coletados foram analisados pelo Índice de Validade de Conteúdo (IVC), considerando-se valores acima de 0,78 e iguais ou maiores que $80 \%$ no Percentual de Concordância ${ }^{20}$.

Utilizou-se um questionário de julgamento ${ }^{21}$, adaptado para avaliação do conteúdo do fluxograma, com dez itens (Tabela 1). Todos os profissionais enfermeiros, médicos e técnicos de enfermagem que participaram da entrevista coletiva (etapa 1), foram convidados a participar da etapa de validação, entretanto, apenas sete profissionais participaram dessa etapa do processo.

Os juízes, então, identificados como profissionais da APS do respectivo município, realizaram o julgamento do fluxograma de atendimento via Google Forms. Procederam à análise, em escala tipo Likert com dois intervalos de respostas, adequado ou não adequado. Ademais, o instrumento constava de espaço para comentários e sugestões. No final da avaliação, as recomendações dos juízes foram acatadas e incorporadas ao fluxograma.

A pesquisa atende a Resolução no 466/12 do Conselho Nacional de Saúde para pesquisas realizadas em seres humanos e foi aprovada pelo Comitê de Ética em Pesquisa sob o parecer no 96023718300000118 .

\section{RESULTADOS}

Os resultados deste estudo são apresentados em duas etapas: produção-construção do fluxograma e validação do fluxograma. 
Tabela 1. Avaliação de concordância do conteúdo do fluxograma. Chapecó-SC, Brasil, 2019.

\begin{tabular}{|c|c|}
\hline Itens avaliados & IVC \\
\hline 1. Utilidade/pertinência: a questão é relevante e atende a finalidade relativa ao procedimento proposto. & 1,0 \\
\hline 2. Consistência: o conteúdo apresenta profundidade suficiente para a compreensão da questão. & 1,0 \\
\hline 3. Clareza: explicitado de forma clara, simples e inequívoca. & 1,0 \\
\hline 4. Objetividade: permite resposta pontual. & 1,0 \\
\hline 5. Simplicidade: a questão expressa uma única ideia. & 1,0 \\
\hline 7. Atualização: as etapas do roteiro seguem as práticas baseadas em evidências mais atuais. & 1,0 \\
\hline 8. Vocabulário: palavras escolhidas adequadamente e sem gerar ambiguidades. & 1,0 \\
\hline 9. Precisão: cada item da avaliação é distinto dos demais, não se confundem. & 0,86 \\
\hline $\begin{array}{l}\text { 10. Sequência instrucional dos tópicos: a sequência da questão se mostra de forma coerente e em ordem } \\
\text { de execuções corretas. }\end{array}$ & 0,86 \\
\hline
\end{tabular}

Fonte: Dados da pesquisa, 2019.

\section{Produção-Construção do fluxograma}

Na primeira etapa, que foi a de construção do fluxograma, foi realizada a coleta de dados, acerca das práticas de saúde desenvolvidas na APS, frente ao indivíduo intoxicado por agrotóxico, e as fragilidades desse atendimento foram apontadas pelos profissionais participantes da pesquisa, por meio de entrevista coletiva. Esse diagnóstico situacional permitiu que o fluxograma se harmonizasse com a realidade e a práxis da equipe de assistência ao paciente.

Durante a entrevista coletiva, os 19 profissionais participantes, sendo, seis enfermeiros, cinco médicos e oito técnicos de enfermagem, todos assistenciais, demonstraram avaliação positiva sobre a existência de um fluxograma, uma vez que referiram ausência desse tipo de tecnologia para auxiliar na assistência ao indivíduo intoxicado por agrotóxico, atendido na APS do município.

Para a construção do conhecimento teórico do fluxograma, utilizou-se conteúdo do Ministério da Saúde, resoluções do Conselho Federal de Enfermagem, portarias, cadernos de estudos e artigos científicos. Em seguida, foram dispostos os símbolos, o conteúdo e as ações a serem realizadas pelos profissionais/ equipes. A primeira versão do fluxograma foi apresentada aos profissionais da APS durante capacitação realizada pela pesquisadora sobre atendimento multiprofissional para casos de intoxicações agudas por agrotóxicos na APS, com o objetivo de coletar sugestões para melhorar o fluxograma. Entretanto, os profissionais fizeram avaliação positiva, não sugerindo modificações. O fluxograma (Figura 1) foi, na sequência, enviado aos juízes para validação do conteúdo.

Também, foi desenvolvido painel de rótulos (Quadro 1), a partir de análise de controle de estoque das empresas com venda autorizada no município, fornecido pela Companhia Integrada de Desenvolvimento Agrícola de Santa Catarina (Cidasc), referente aos agrotóxicos mais comercializados no segundo semestre de 2017 e primeiro semestre de 2018 . O painel contém classificação dos agrotóxicos segundo o modo de ação, princípio ativo, nome comercial, classe toxicológica e manifestações clínicas agudas, decorrentes da exposição aos mesmos. No painel, as cores representam o grau de toxicidade de cada produto, sendo a faixa vermelha extremamente tóxico, faixa amarela altamente tóxico, faixa azul medianamente tóxico e faixa verde pouco tóxico. O painel de rótulos foi construído para orientar os profissionais quanto ao tipo de agrotóxico que o usuário possa ter tido contato e facilitar a conduta, sendo um instrumento anexo ao fluxograma.

\section{Validação do fluxograma}

Nesta etapa, o fluxograma foi avaliado por sete juízes, sendo eles: três enfermeiras do sexo feminino; um médico do sexo masculino; uma médica do sexo feminino; e duas técnicas de enfermagem do sexo feminino, atuantes na APS do respectivo município, sendo esse número, considerado representativo para validação do conteúdo ${ }^{17}$. A idade dos juízes variou de 29 a 55 anos (média de 38 anos e Desvio Padrão (DP) de 8,2). Dos sete juízes, quatro $(57,1 \%)$ tinham título de especialista em saúde da família e um de mestre nessa área.

No processo de validação do fluxograma, considerando cada item, separadamente, a concordância entre os juízes quanto à utilidade, consistência, clareza, objetividade, simplicidade, exequibilidade, atualização e vocabulário foi de $100 \%$, recebendo IVC de valor máximo (IVC 1,0). Em contrapartida, nos itens precisão e sequência instrucional dos tópicos, obteve-se índices diferentes (0,86 em cada item), o que não comprometeu os resultados, pois o valor está acima do limite desejado (Tabela 1). O IVC global foi igual a 0,97, dessa maneira, não foi necessário submeter o instrumento a uma nova avaliação.

Os juízes avaliaram de forma positiva os itens relacionados ao conteúdo do fluxograma, entretanto, fizeram sugestões para aprimorá-lo. As alterações sugeridas foram analisadas pela pesquisadora, com base em literatura pertinente, e acatadas conforme o Quadro 2, a seguir. 


\section{FLUXOGRAMA ${ }^{19}$ DE ATENDIMENTO MULTIPROFISSIONAL PARA CASOS DE INTOXICAÇÃO AGUDA POR AGROTÓXICOS NAATENÇÃO PRIMÁRIA À SAÚDE}

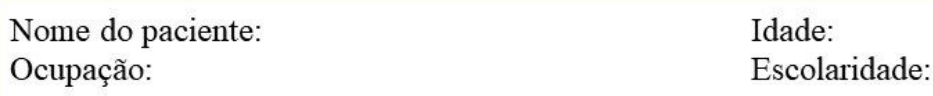

Idade:

Escolaridade:

Figura 1. Fluxograma ${ }^{19}$ de atendimento multiprofissional para casos de intoxicações agudas por agrotóxicos na Atenção Primária à Saúde ${ }^{22,23}$. Legenda: * Consulta de Enfermagem/avaliação realizado pelo Enfermeiro, com as informações registrada via sistema IPM saúde. ** Painel de rótulos desenvolvido pela autora, referente aos agrotóxicos mais comercializados em Itapiranga-SC no segundo semestre/2017 e primeiro semestre/2018. ${ }^{* * *}$ Em caso de dúvida o profissional poderá contatar o Centro de Informação e Assistência Toxicológica de Santa Catarina CIATox através do número 08006435252 . **** Agentes Comunitários de Saúde (ACS) auxiliam na identificação de áreas de utilização de produtos químicos. Serviços de urgência e emergência: Sociedade Hospitalar Itapiranga Ltda no: 4936770141; SAMU no: 192, Corpo de Bombeiros no: 193. Serviço de transporte da Secretaria Municipal de Saúde de Itapiranga-SC no: 49367877743. Fonte: Elaborado pelas autoras, 2019. 


\section{Fluxograma para intoxicações por agrotóxicos}

Karal A, Portaluppi DM, Zocche DAA, Zanatta L

Quadro 1. Painel de rótulos* dos agrotóxicos mais comercializados nos semestres 02/2017 e 01/2018 no município de pesquisa ${ }^{24-27}$. Legenda: *Companhia Integrada de Desenvolvimento Agrícola de Santa Catarina CIDASC - Departamento Regional de São Miguel do Oeste forneceu tabela de controle de estoque de agrotóxicos das empresas com venda autorizada no município de Itapiranga.

\section{Painel de Rótulos*}



Fonte: Elaborado pelas autoras, 2019. 
Quadro 2. Modificações realizadas no fluxograma segundo sugestões dos juízes.

\begin{tabular}{|c|c|}
\hline Sugestões dos juízes & Modificações realizadas \\
\hline \multirow[b]{2}{*}{ Inserir verificação de sinais vitais (E1) } & $\begin{array}{l}\text { Inserido cabeçalho com: nome do paciente, idade, } \\
\text { escolaridade e ocupação. }\end{array}$ \\
\hline & $\begin{array}{l}\text { Modificado terminal de "Paciente com sinais e sintomas de } \\
\text { Intoxicação Aguda por Agrotóxico (IAA) recebido na Atenção } \\
\text { Primária à Saúde (APS)" para "Acolhe e verifica SSVV". }\end{array}$ \\
\hline \multirow{4}{*}{$\begin{array}{l}\text { Inserir avaliação do paciente por enfermeiro e técnico de } \\
\text { enfermagem (E2) }\end{array}$} & $\begin{array}{l}\text { Substituído processo "Acolhimento e consulta de } \\
\text { enfermagem e médica" para "Encaminha para enfermeiro } \\
\text { assistencial" e para "Classifica o risco e realiza CE". }\end{array}$ \\
\hline & $\begin{array}{l}\text { Inserido processo “Identifica algum dos sinais e sintomas } \\
\text { descritos. }\end{array}$ \\
\hline & $\begin{array}{l}\text { Inserido ponto de decisão: "Teve contato com algum } \\
\text { produto químico nas últimas } 24 \text { horas?" com as alternativas } \\
\text { "Não" e terminal "Paciente segue fluxo da APS", ou "Sim" } \\
\text { e processo "Identifica o tipo de contato e relata o tipo de } \\
\text { produto que teve contato". }\end{array}$ \\
\hline & $\begin{array}{l}\text { Substituir processo "Se suspeitar de IAA: } 1 \text { ㅇ Notificar. *C 2ㅇ } \\
\text { Estratificar risco" para "Se considerar suspeita de intoxicação } \\
\text { por agrotóxicos, notificar (Sinan)". }\end{array}$ \\
\hline $\begin{array}{l}\text { Inserir necessidade de encaminhamento para atendimento } \\
\text { médico (E1) }\end{array}$ & Inserido processo "Encaminha para equipe médica". \\
\hline \multirow{4}{*}{ Reformular fluxograma para agilizar e otimizar (M1) } & $\begin{array}{l}\text { Substituído frase em ponto de decisão de "É diagnóstico de } \\
\text { IAA?", para "Diagnóstico de IAA?". }\end{array}$ \\
\hline & Substituído terminal "A", para "Paciente segue fluxo da APS". \\
\hline & $\begin{array}{l}\text { Frase modificada de "Estratificação de risco", para } \\
\text { "Gravidade da intoxicação". }\end{array}$ \\
\hline & $\begin{array}{l}\text { Retirado caixa de texto com os profissionais envolvidos e } \\
\text { dispostos dentro dos símbolos. }\end{array}$ \\
\hline
\end{tabular}

Fonte: Dados da pesquisa, 2019.

Desse modo, após validação e realização das mudanças sugeridas, obteve-se a versão final do fluxograma (Figura 1) que, posteriormente, passou por aprovação no Conselho Municipal de Saúde do município em questão, com registro em ata. $O$ instrumento apresentado teve avaliação positiva, enquanto tecnologia para melhorar a assistência ao indivíduo intoxicado por agrotóxico, bem como foi mencionada a relevância do fluxograma para melhorar as notificações de intoxicação por agrotóxico no município.

\section{DISCUSSÕES}

O desenvolvimento do fluxograma proposto surgiu em meio à necessidade de melhorar a identificação dos casos de intoxicação por agrotóxico na APS de um município do ExtremoOeste do estado de Santa Catarina, uma vez que estimativas da Organização Mundial da Saúde (OMS) apontam que, para cada evento de intoxicação notificado, existem 50 não notificados ${ }^{8}$. Nesta pesquisa, foram abordados os casos agudos de intoxicação por agrotóxico, em virtude dos sintomas, geralmente, apresentarem características inespecíficas, o que leva a dificuldades em estabelecer diagnóstico e, portanto, contribui para subnotificação dos $\operatorname{casos}^{28}$.

A elaboração do fluxograma vem suprir a necessidade de qualificar o atendimento prestado ao indivíduo intoxicado por agrotóxico, por meio de uma tecnologia facilitadora e norteadora da assistência. Ainda, a elaboração desse fluxograma pode ser considerada um avanço para a assistência dos casos de intoxicação por agrotóxico no município e região, cuja economia gira em torno do agronegócio, além de ser de baixo custo.

A busca da literatura disponível, aliada ao conhecimento das práticas de saúde e as fragilidades identificadas no atendimento ao indivíduo intoxicado por agrotóxico, relatadas pelos profissionais da APS, foi fundamental para que o fluxograma fosse construído, 
levando em conta o contexto real das equipes e que pudesse ser harmonizado com a práxis profissional. Pensando na aplicabilidade da tecnologia construída, foi elaborado um painel de rótulos, visando auxiliar os profissionais na identificação do tipo de agrotóxico que causou a intoxicação para, dessa forma, agilizar e garantir resolubilidade na conduta. Esse painel incorpora o fluxograma.

Nesse contexto, uma vez que os profissionais da saúde precisam estar capacitados para identificar, fazer o diagnóstico e tratar os casos com ênfase na promoção, na educação em saúde e na prevenção de danos ${ }^{8,29}$, a tecnologia construída emerge como facilitadora desse processo. E não somente, mas como uma aliada às diversas ações que promovem qualidade de vida e saúde dos indivíduos, e que consideram os diversos determinantes do processo saúde-doença, de suma importância para a criação de ambientes saudáveis e de proteção do meio ambiente, bem como reorientação dos serviços de saúde que coadunam às ideias e evidências levantadas nas conferências para a promoção da saúde.

A percepção de riscos e as ações de prevenção, promoção e educação em saúde são atributos essenciais e inerentes aos serviços da APS ${ }^{11}$. Difundir conhecimento acerca da temática é primordial para a sensibilização quanto ao uso adequado dos equipamentos de proteção, sejam eles individuais ou coletivos, bem como para o reconhecimento e associação de sinais e sintomas com o uso de agrotóxicos, favorecendo a identificação e a conduta adequada frente aos casos identificados.

O fluxograma pode subsidiar novas possibilidades, ações para mudanças de comportamento, de modo a agregar promoção à saúde e prevenção de agravos ${ }^{30}$. Sinaliza para elaboração de ações frente à exposição e uso de medidas protetivas, de modo a minimizar os riscos de exposição aos agrotóxicos. Para tanto, os trabalhadores da saúde necessitam realizar atividades de vigilância epidemiológica e implementar estratégias de atenção à saúde dos indivíduos, seja capacitando equipes de saúde ou grupos na comunidade e, em especial, aos trabalhadores rurais ${ }^{31}$.

Entretanto, estudos apontam que o indivíduo estar ciente dos riscos devido ao uso de agrotóxicos, não é suficiente para provocar mudanças de conduta, necessitando de outros mecanismos de cumprimento das normas de segurança e de políticas de controle ${ }^{31}$, que podem ser articuladas com as diferentes esferas de governo e setores, na intenção de trazer o assunto da saúde a todas as políticas públicas.

A escolha dos profissionais que atuam na APS do respectivo município, para a composição dos juízes, foi realizada tendo em vista a capacidade de esses profissionais identificarem o fluxo de trabalho, e a percepção de que a participação ativa da equipe multiprofissional é primordial para que o instrumento seja utilizado e fortaleça a qualidade assistencial ${ }^{32}$.

No processo de análise de conteúdo, as contribuições dos juízes foram incluídas, uma vez que surgiram informações relevantes de fluxo e ações, não apresentadas anteriormente. Os juízes julgaram o fluxograma apto para aplicabilidade na prática clínica profissional (IVC 1,0). Nessa perspectiva, a elaboração do fluxograma representa uma tecnologia que direciona os trabalhadores e contribui para a assistência ${ }^{16}$.

Com relação aos tipos de instrumentos utilizados para avaliação do fluxograma, escores de um teste permitem ao pesquisador analisar a exatidão de determinada inferência, o qual permeia todo o processo de construção, aplicação, correção e resultados, com a intenção de verificar se o conteúdo proposto responde a todos os aspetos do objeto ${ }^{17}$. A utilização de um questionário de julgamento eletrônico, por meio da ferramenta Google Forms mostrou-se de fácil aplicação, facilitando a coleta de dados para validação, bem como a visualização dos resultados individualizados ou não.

Nesta pesquisa, foi apresentado apenas uma vez o fluxograma para validação, pois na análise quantitativa, os dez índices foram considerados satisfatórios, IVC $\geq 0,86$ e global de 0,97. Portanto, considera-se validado o Fluxograma de atendimento multiprofissional para casos de intoxicação aguda por agrotóxicos na Atenção Primária à Saúde. Estudos de validação de tecnologias cuidativo-educacionais e assistenciais costumam utilizar o IVC para validar o conteúdo do produto tecnológico desenvolvido, bem como a realização de ajustes até a versão final validada ${ }^{32,33}$.

O estudo não foi validado quanto a aparência. Pode apresentar limitações, em virtude de ter sido realizado para a prática profissional da APS de uma determinada região e sua aplicabilidade em outras regiões do estado ou País podem necessitar de adaptações. Além disso, trata-se de uma tecnologia direcionada ao diagnóstico de casos de intoxicações agudas por agrotóxicos, não sendo aplicado aos casos crônicos. Para tanto, sugere-se novos estudos que abordem as características das intoxicações crônicas.

\section{CONSIDERAÇÕES FINAIS E IMPLICAÇÕES PARA A PRÁTICA}

Neste trabalho, apresentou-se o processo de produçãoconstrução e validação de um fluxograma multiprofissional para atendimento de intoxicações agudas por agrotóxicos na APS. $\mathrm{O}$ desenvolvimento desta tecnologia, além de ser baseado no conhecimento científico, também levou em consideração às necessidades identificadas pelos próprios profissionais da APS, para que fosse possível a produção-construção de uma ferramenta de trabalho aplicável à realidade.

Entende-se que, por constituir um instrumento de orientação da prática, o fluxograma multiprofissional para atendimento de intoxicações agudas por agrotóxicos contribui para a qualificação profissional, instrumentalizando o profissional da APS frente a esse atendimento e qualificando a assistência ao usuário do serviço. Além disso, mostra-se como tecnologia transformadora do processo de trabalho para otimizar, organizar e qualificar a assistência, auxiliando na investigação, identificação e direcionamento dos casos suspeitos ou confirmados de intoxicações agudas por agrotóxicos na APS. Para a enfermagem e saúde, em geral, a tecnologia aqui apresentada também se revela como uma ferramenta de gestão do cuidado ao indivíduo intoxicado por agrotóxicos, permitindo a sistematização da assistência e, consequente, qualidade desse atendimento. 
Ademais, essa tecnologia apresenta-se como instrumento inovador de pesquisa e ensino, podendo ser empregada no ensino da assistência às intoxicações agudas por agrotóxicos. Outrossim, os resultados desse estudo propiciam conhecimentos acerca das práticas de saúde e necessidades frente as intoxicações agudas por agrotóxicos, o que possibilita capacitação em saúde, divulgação do tema pesquisado, além de abrir portas para novas pesquisas nessa área.

Sobre as limitações do estudo, configura-se importante frente ao reduzido número de estudos atuais que abordam essa temática, especificamente, que apresentam instrumentos para os profissionais da APS utilizarem na assistência ao indivíduo intoxicado por agrotóxico. Também, pelo restrito número de juízes que participaram do estudo e por serem profissionais apenas da APS do município em que a pesquisa foi realizada, portanto, os resultados não podem ser estendidos a outras regiões geográficas e a outros níveis de atenção à saúde.

No intuito de aperfeiçoar essa tecnologia, recomenda-se a realização de validação por especialistas na área de toxicologia clínica. Considera-se, também, a necessidade de contínua atualização, além da avaliação de sua aplicabilidade no cenário apresentado, bem como em outros níveis de atenção à saúde.

\section{FINANCIAMENTO}

Fapesc TO 2021TR808.

\section{CONTRIBUIÇÕES DOS AUTORES}

Desenho do estudo. Adriane Karal. Leila Zanatta. Denise Antunes de Azambuja Zocche

Coleta ou produção dos dados. Adriane Karal. Leila Zanatta. Denise Antunes de Azambuja Zocche

Análise de dados. Adriane Karal. Dara Montag Portaluppi. Leila Zanatta. Denise Antunes de Azambuja Zocche

Interpretação dos resultados. Adriane Karal. Dara Montag

Portaluppi. Leila Zanatta. Denise Antunes de Azambuja Zocche

Redação e revisão crítica do manuscrito. Adriane Karal. Dara Montag Portaluppi. Leila Zanatta. Denise Antunes de Azambuja Zocche

Aprovação da versão final do artigo. Adriane Karal. Dara Montag Portaluppi. Leila Zanatta. Denise Antunes de Azambuja Zocche

Responsabilidade por todos os aspectos do conteúdo e a integridade do artigo publicado. Adriane Karal. Dara Montag Portaluppi. Leila Zanatta. Denise Antunes de Azambuja Zocche

\section{EDITOR ASSOCIADO}

Candida Primo Caniçali $₫$

\section{EDITOR CIENTÍFICO}

Ivone Evangelista Cabral (1)

\section{REFERÊNCIAS}

1. World Health Organization. The Ottawa Charter for Health Promotion [Internet]. Geneva: WHO; 2020 [citado 2020 ago 2]. Disponível em: www.who.int/teams/health-promotion/enhanced-wellbeing/first-globalconference

2. World Health Organization. The 8th Global Conference on Health Promotion [Internet]. Geneva:WHO; 2021 [citado 2021 maio 4]. Helsinki, Finland 10-14 June 2013. Disponível em: https://www.who.int/healthpromotion/ conferences/8gchp/8gchp_helsinki_statement.pdf

3. World Health Organization. Shanghai Declaration on promoting health in the 2030 Agenda for Sustainable Development [Internet]. Geneva: WHO; 2021 [citado 2021 maio 4]. Disponível em: https://www.who.int/ news/item/07-11-2016-shanghai-declaration-on-promoting-health-inthe-2030-agenda-for-sustainable-development

4. Camponogara S, De Lima Rodrigues IL, Dias GL, Moura LN, Viero CM, Miorin JD. Implications of pesticide use: perceptions of families of children with cancer. Rev Pesqui Cuid Fundam. 2017 jul/set;9(3):786-94 http://dx.doi.org/10.9789/2175-5361.2017.v9i3.786-794.

5. Ministério da Saúde (BR). Secretaria de Vigilância em Saúde. Diretrizes nacionais para a vigilância em saúde de populações expostas a agrotóxicos [Internet]. Brasília: MS; 2017 [citado 2020 ago 2]. Disponíve em: https://bvsms.saude.gov.br/bvs/publicacoes/diretrizes_vigilancia_ populacoes_expostas_agrotoxicos.pdf

6. Paumgartten FJR. Pesticides and public health in Brazil. Curr Opin Toxicol. 2020 jan;22:7-11. http://dx.doi.org/10.1016/j.cotox.2020.01.003.

7. Friedrich K, Soares VE, Da Silva Augusto LG, Gurgel AM, De Souza MMO Alexandre VP et al. Agrotóxicos: mais venenos em tempos de retrocessos de direitos. Revista OKARA: Geografia em debate. 2018;12(2):326-47. http://dx.doi.org/10.22478/ufpb.1982-3878.2018v12n2.41320.

8. Carneiro FF, Augusto LGS, Rigotto RM, Friedch K, Búrigo AC organizadores. Dossiê ABRASCO: um alerta sobre os impactos dos agrotóxicos na saúde [Internet]. Rio de Janeiro: EPSJV; São Paulo: Expressão Popular; 2015 [citado 2020 ago 2]. Disponível em: https:// www.abrasco.org.br/dossieagrotoxicos/wpcontent/uploads/2013/10/ DossieAbrasco_2015_web.pdf

9. Nações Unidas Brasil. Objetivo de Desenvolvimento Sustentável 12 Consumo e produção responsáveis [Internet]. Brasília: Nações Unidades; 2021 [citado 2021 maio 4]. Disponível em: https://brasil.un.org/index. php/pt-br/sdgs/12

10. Ministério da Saúde (BR). Relatório Nacional de Vigilância em Saúde de Populações Expostas a Agrotóxicos [Internet]. v. 2. Brasília: MS 2018 [citado 2020 ago 2]. Disponível em: https://bvsms.saude.gov.br/ bvs/publicacoes/relatorio_nacional_vigilancia_populacoes_expostas agrotoxicos.pdf

11. Portaria $\mathrm{n}^{\circ} \mathbf{2 . 4 3 6}$, de 21 de setembro de 2017 (BR). Aprova a Política Nacional de Atenção Básica, estabelecendo a revisão de diretrizes para a organização da Atenção Básica, no âmbito do Sistema Único de Saúde (SUS). Diário Oficial da União [periódico na internet], Brasília (DF), 22 set 2017 [citado 8 jan. 2020]. Disponível em: https://www. jusbrasil.com.br/diarios/161636273/dou-secao-1-22-09-2017-pg-68

12. Melo WF, Melo CFP, Saldanha HGAC, Rodrigues LMS. Assistência de enfermagem à vítima de intoxicação exógena. REBES. [Internet]. 2015 abr/jun [citado 2020 ago 2];5(2):[aprox. 8 telas]. Disponível em: www. gvaa.com.br/revista/index.php/REBES/article/view/4020/4180

13. Cargnin MCS, Echer IC, Silva DR. Tobacco farming: use of personal protective equipment and pesticide poisoning. Rev Pesqui Cuid Fundam. 2017 abr/jun;9(2):466-72. http://dx.doi.org/10.9789/2175-5361.2017. v9i2.466-472.

14. Silva JCS, Coelho MJ, Pinto CMI. Factors associated with the deaths of men poisoned by carbamato ("chumbinho"). Rev Gaúcha Enferm. 2016 jun;37(2):e54799. http://dx.doi.org/10.1590/1983-1447.2016.02.54799. PMid:27253592.

15. Costa NP, Polaro SHI, Vahl EAC, Gonçalves LHT. Storytelling: a care technology in continuing education for active ageing. Rev Bras Enferm. 2016 nov/dez;69(6):1132-39. http://dx.doi.org/10.1590/0034-71672016-0390. PMid:27925090.

16. de Aquino MST, Neto PHS, Dutra CS, Vasconcelos PF. Implementation of a healthcare workflow in a psychosocial care center. Rev Bras Promoç 
Saúde. 2017 abr/jun;30(2):288-93. http://dx.doi.org/10.5020/18061230.2017. p288.

17. Marinho PML, Campos MPA, Rodrigues EOL, Gois CFL, Barreto IDC. Construction and validation of a tool to Assess the Use of Light Technologies at Intensive Care Units. Rev Lat Am Enfermagem. 2016 dec;24:e2816. http://dx.doi.org/10.1590/1518-8345.1002.2816.

18. Polit DF, Beck CT. Fundamentos de pesquisa em enfermagem: avaliação de evidências para as práticas da enfermagem. 7. ed. Porto Alegre: Artmed; 2011.

19. Silva GD, Silvino ZR. Elaboração de fluxogramas. In: Silvino ZR, organizador. Gestão baseada em evidências: recursos inteligentes para soluções de problemas na prática em saúde. Curitiba: CRV; 2018. cap. 5 .

20. Alexandre NMC, Coluci MZO. Content validity in the development and adaptation processes of measurement instruments. Cien Saude Colet. 2011 jul;16(7):3061-8. http://dx.doi.org/10.1590/S1413-81232011000800006. PMid:21808894.

21. Tibúrcio MP, Melo GSM, Balduíno LSC, Costa IKF, Dias TYAF, Torres GV. Validação de instrumento para avaliação da habilidade de mensuração da pressão arterial. Rev Bras Enferm. 2014 jul/ago;67(4):581-7. http:// dx.doi.org/10.1590/0034-7167.2014670413. PMid:25271583.

22. Resolução COFEN 661/2021. (BR). Atualiza e normatiza, no âmbito do Sistema Cofen/Conselhos Regionais de Enfermagem, a participação da Equipe de Enfermagem na atividade de Classificação de Risco. Diário Oficial da União [periódico na internet], Brasília (DF), 9 mar 2021 [citado 8 ago. 2021]. Disponível em: http://www.cofen.gov.br/ resolucao-cofen-no-661-2021_85839.html

23. Portaria $n^{\circ} 43$, de 16 de outubro de 2018. (BR). Torna pública a decisão de aprovar as Diretrizes Brasileiras para diagnóstico e tratamento das intoxicações por agrotóxicos - capítulo 1, no âmbito do Sistema Único de Saúde - SUS. Diário Oficial da União [periódico na internet], Brasília (DF), 16 out 2018 [citado 2020 jan 8]. Disponível em: https://www.in.gov. br/materia/-/asset_publisher/Kujrw0TZC2Mb/content/id/45778984/ DiarioOficialdaUniao

24. Ministério da Saúde (BR). Monografias autorizadas [Internet]. Brasília: MS; 2021 [citado 2021 ago 2]. Disponível em: https://www.gov.br/ anvisa/pt-br/setorregulado/regularizacao/agrotoxicos/monografias/ monografias-autorizadas-por-letra
25. Vigilância Sanitária de Santa Catarina. (BR). Intoxicações por agrotóxicos: informações básicas para o manejo clínico [Internet] Florianópolis: Secretaria de Estado da Saúde Santa Catarina [citado 2020 Aug 2]. Disponível em: http://www.vigilanciasanitaria.sc.gov.br/ phocadownload/Noticias/2018/Novembro/cartaz_agrotoxicos\%20 ministrio\%20pblicoedivs.pdf

26. Agrolink. Agrolinkfito [Internet]. Porto Alegre: Agrolink; 2021 [citado 2020 ago 2]. Disponível em: https://www.agrolink.com.br/agrolinkfito/ busca-direta-produto

27. Agro Bayer Brasil. Produtos para a proteção de cultivos [Internet]. São Paulo: Agro Bayer Brasil; 2020 [citado 2020 ago 2]. Disponível em: https:// www.agro.bayer.com.br/essenciais-do-campo/protecaodecultivos

28. Taveira BLS, Albuquerque GSC. Análise das notificações de intoxicações agudas, por agrotóxicos, em 38 municípios do estado do Paraná. Saúde Debate. 2018 dez;42(esp 4):211-22. http://dx.doi.org/10.1590/010311042018 s417.

29. Viero CM, Camponogara S, Cezar-Vaz MR, Costa VZ, Beck CLC. Risk society: the use of pesticides and implications for the health of rura workers. Esc Anna Nery. 2016 jan/mar;20(1):99-105. http://dx.doi. org/10.5935/1414-8145.20160014.

30. Carvalho FFB, Cohen SC, Akerman M. Refletindo sobre o instituído na Promoção da Saúde para problematizar 'dogmas'. Saúde Debate. 2017;41(spe 3):265-76. http://dx.doi.org/10.1590/0103-11042017s320.

31. Martins VHS, Carvalho MAB, Belfort LRM, Guisande TCCA, Santos TV. The role of work nursing in the prevention of the risks of workers exposed to pesticides: a bibliographical review. Res Soc Dev. 2019 mar 29;8(6):e19861039. http://dx.doi.org/10.33448/rsd-v8i6.1039.

32. Moura IH, Silva AFR, Rocha AESH, Lima LHO, Moreira TMM, Silva ARV. Construction and validation of educational materials for the prevention of metabolic syndrome in adolescents. Rev Lat Am Enfermagem. 2017;25:e2934. http://dx.doi.org/10.1590/1518-8345.2024.2934. PMid:29020125.

33. Lima ACMACC, Bezerra KC, Sousa DMN, Rocha JF, Oriá MOB Development and validation of a booklet for prevention of vertical HIV transmission. Acta Paul Enferm. 2017;30(2):181-9. http://dx.doi. org/10.1590/1982-0194201700028. 